\title{
Dot product representations of planar graphs
}

\author{
Ross J. Kang* \\ Durham University \\ ross.kang@gmail.com \\ Tobias Müller \\ Centrum Wiskunde \& Informatica \\ tobias@cwi.nl \\ László Lovász \\ Eötvös Loránd University \\ lovasz@cs.elte.hu \\ Edward R. Scheinerman \\ The John Hopkins University \\ ers@jhu.edu \\ Submitted: Oct 23, 2010; Accepted: Oct 28, 2011; Published: Nov 7, 2011 \\ Mathematics Subject Classification: $05 \mathrm{C} 62$
}

\begin{abstract}
A graph $G$ is a $k$-dot product graph if there exists a vector labelling $u: V(G) \rightarrow$ $\mathbb{R}^{k}$ such that $u(i)^{\mathrm{T}} u(j) \geq 1$ if and only if $i j \in E(G)$. Fiduccia, Scheinerman, Trenk and Zito [Discrete Math., 1998] asked whether every planar graph is a 3-dot product graph. We show that the answer is "no". On the other hand, every planar graph is a 4-dot product graph. We also answer the corresponding questions for planar graphs of prescribed girth and for outerplanar graphs.
\end{abstract}

\section{Introduction and statement of results}

We study a type of geometric representation of graphs using vectors from $\mathbb{R}^{k}$ for some $k \in \mathbb{N}$. Let $G$ be a graph. We say $G$ is a $k$-dot product graph if there exists a vector labelling $u: V(G) \rightarrow \mathbb{R}^{k}$ such that $u(i)^{\mathrm{T}} u(j) \geq 1$ if and only if $i j \in E(G)$. An explicit set of vectors in $\mathbb{R}^{k}$ that exhibits $G$ in this way is called a $k$-dot product representation of $G$. The dot product dimension of $G$ is the least $k$ such that there is a $k$-dot product representation of $G$. Every graph has finite dot product dimension; see for instance [4].

The well-studied class of threshold graphs is closely related to 1-dot product graphs: a 1-dot product graph has at most two nontrivial connected components and each of these

\footnotetext{
${ }^{*}$ Research partially supported by the Engineering and Physical Sciences Research Council (EPSRC), grant EP/G066604/1.

${ }^{\dagger}$ Research supported by the European Research Council (ERC), grant 227701.

${ }^{\ddagger}$ Research partially supported by a VENI grant from the Netherlands Organisation for Scientific Research (NWO).
} 
components is a threshold graph (cf. [4]). Mahadev and Peled [9] give an extensive survey of threshold graphs.

Notions closely related to dot product representations were studied in the context of communications complexity by amongst others Paturi and Simon [10], Alon, Frankl and Rödl [1] and Lovász [8]. Partially motivated by these treatments, Reiterman, Rödl and Šiňajová $[11,12,13]$ introduced dot product representations of graphs and studied them extensively. They obtained several bounds for the dot product dimension in terms of threshold dimension, sphericity, chromatic number, maximum degree, maximum average degree, and maximum complementary degree; they also detailed various examples. Here it should be mentioned that they used a slightly different definition: they used a threshold $t \in \mathbb{R}$ (i.e. $i j \in E(G)$ if and only if $u(i)^{\mathrm{T}} u(j) \geq t$ ). This leads to a slightly larger class of graphs. However, in most of their constructions, Reiterman et al. in fact take $t=1$. The proofs of our results below can easily be adapted to work also for the more general definition of a dot product graph used in [11, 12, 13]. Fiduccia, Scheinerman, Trenk and Zito [4] considered amongst other things the dot product dimension of bipartite, complete multipartite and interval graphs.

Both Reiterman et al. [12] and Fiduccia et al. [4] proved that every forest is a 3-dot product graph, and there exist trees which are not 2-dot product graphs. Envisioning a potential extension to this result, Fiduccia et al. asked whether every planar graph is a 3-dot product graph. Here we will answer this question in the negative by describing two counterexamples. In contrast, any planar graph has dot product dimension at most 4 .

Theorem 1 Every planar graph is a 4-dot product graph, and there exist planar graphs that are not 3-dot product graphs.

In Section 3, we show that every planar graph has a 4-dot product representation. In Section 4, we present one small example (on eighteen vertices) of a planar graph that is not a 3-dot product graph. It remains open to characterise which planar graphs have dot product dimension exactly 4; however, we do demonstrate the following.

Theorem 2 Every planar graph of girth at least 5 is a 3-dot product graph, and there exist planar graphs of girth 4 that are not 3-dot product graphs.

We exhibit a planar graph of girth 4 that has dot product dimension 4 in Section 5 . In the next section, we develop some notation and recollect some spherical geometry needed in Sections 3 and 5.

Let us also mention that as a direct consequence of a result of Kotlov, Lovász and Vempala [7] (repeated as Theorem 7 below) every outerplanar graph has dot product dimension at most 3. Given the examples of trees that are not 2-dot product graphs given by Fiduccia et al. [4], we have the following.

Theorem 3 Every outerplanar graph is a 3-dot product graph, and there are outerplanar graphs that are not 2-dot product graphs.

Some of the results of this paper were presented at the 18th International Symposium on Graph Drawing (GD 2010) in Konstanz [5]. 


\section{Preliminaries}

Let $S^{k}$ denote the unit sphere in $\mathbb{R}^{k+1}$, i.e. $S^{k}=\left\{u \in \mathbb{R}^{k+1}:\|u\|=1\right\}$. Let us review some basic geometry on $S^{k}$. For $u, v \in S^{k}$, let us denote by $[u, v]$ the (shortest) spherical arc between $u$ and $v$. Let $\operatorname{dist}_{S^{k}}(u, v)$ denote the length of $[u, v]$. Then $\operatorname{dist}_{S^{k}}(u, v)$ equals the angle between the two vectors $u, v \in S^{k}$. It can thus be expressed as

$$
\operatorname{dist}_{S^{k}}(u, v)=\arccos \left(v^{\mathrm{T}} u\right) .
$$

For $0<r<\pi$, let the spherical cap of radius $r$ around $v$ on $S^{k}$ be defined as

$$
\operatorname{cap}_{S^{k}}(v, r):=\left\{u \in S^{k}: \operatorname{dist}_{S^{k}}(u, v) \leq r\right\} .
$$

For $r<\pi / 2$, a spherical cap can also be written in terms of a fixed vector $w \in \mathbb{R}^{k+1}$ with $\|w\|>1$ :

$$
\operatorname{cap}_{S^{k}}(v, r)=\operatorname{cap}_{S^{k}}(w):=\left\{u \in S^{k}: w^{\mathrm{T}} u \geq 1\right\}
$$

The vector $w$ and the radius $r$ of the spherical cap are related via $\|w\|=1 / \cos (r)$ (remembering that $0<r<\pi / 2$ ). We will mostly work in $S^{2}$, in which case we drop the subscript and simply use dist and cap.

Suppose that $u, v, w \in S^{2}$ are three points on the sphere in general position (i.e. not all three on the same great circle/equator). We shall call the union of the three spherical $\operatorname{arcs}[u, v],[v, w],[u, w]$ a spherical triangle. Let us write $a:=\operatorname{dist}(u, v), b:=\operatorname{dist}(u, w)$, $c:=\operatorname{dist}(v, w)$, and let $\gamma$ denote the angle between $[u, v]$ and $[u, w]$. See Figure 1. Recall the spherical law of cosines:

$$
\cos (c)=\cos (a) \cos (b)+\sin (a) \sin (b) \cos (\gamma) .
$$

The spherical law of cosines can be rephrased as

$$
v^{\mathrm{T}} w=\left(u^{\mathrm{T}} v\right) \cdot\left(u^{\mathrm{T}} w\right)+\cos (\gamma) \sqrt{\left(1-\left(u^{\mathrm{T}} v\right)^{2}\right)\left(1-\left(u^{\mathrm{T}} w\right)^{2}\right)}
$$

This second form will be more useful for our purposes.

Similarly to a spherical triangle, one can define a spherical n-gon determined by $u_{1}, \ldots, u_{n} \in S^{k}$ as $\bigcup_{i=1}^{n}\left[u_{i}, u_{i+1}\right]$ (where $u_{n+1}:=u_{0}$ ). In the proof of Theorem 8, we will make use of the well-known fact that the sum of the angles of a spherical $n$-gon is strictly larger than $(n-2) \pi$.

\section{All planar graphs are 4-dot product graphs}

We present a short argument that the dot product dimension of every planar graph is at most 4, one that makes use of Koebe's Theorem [6].

Given a graph $G$, we say that a vector labelling $u: V(G) \rightarrow \mathbb{R}^{k}$ is a cap contact representation of $G$ on $S^{k}$ if it satisfies the following: 


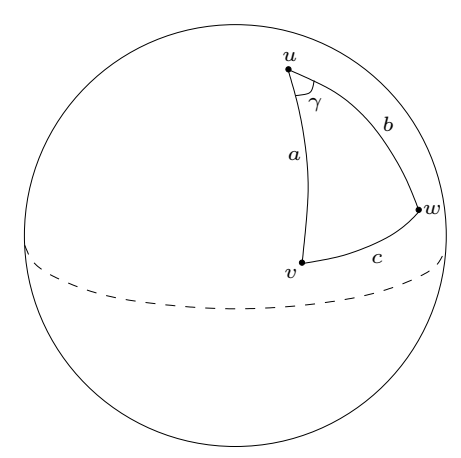

Figure 1: A spherical triangle.

(i) $\operatorname{cap}_{S^{k}}(u(i)) \cap \operatorname{cap}_{S^{k}}(u(j))$ is a singleton if $i j \in E(G)$;

(ii) $\operatorname{cap}_{S^{k}}(u(i)) \cap \operatorname{cap}_{S^{k}}(u(j))$ is empty if $i j \notin E(G)$.

In terms of cap contact representations, Koebe's Theorem (originally given in terms of circle packings in the plane) can be reformulated as follows.

Theorem 4 (Koebe [6]) A graph is planar if and only if it has a cap contact representation on $S^{2}$.

Proposition 5 If a graph $G$ has a cap contact representation on $S^{k}$, then $G$ is a $(k+2)$ dot product graph.

Proof: For $w \in \mathbb{R}^{k}$ with $\|w\|>1$, let us denote by $\theta(w)$ the $\operatorname{radius~of~} \operatorname{cap}_{S^{k}}(w)$, i.e. $\theta(w)=$ $\arccos (1 /\|w\|)$.

Suppose $u: V(G) \rightarrow \mathbb{R}^{k}$ is a cap contact representation of $G$ on $S^{k}$. Let us define $f: V(G) \rightarrow \mathbb{R}^{k}$ by setting $f(i):=(u(i), \tan (\theta(u(i))))^{T}$. We now demonstrate that $f$ is a $(k+2)$-dot representation of $G$.

If $i j \in E(G)$, then the angle between $u(i)$ and $u(j)$ is precisely $\theta(u(i))+\theta(u(j))$. Thus

$$
\begin{array}{rl}
f(i)^{\mathrm{T}} & f(j)=u(i)^{\mathrm{T}} u(j)+\tan (\theta(u(i))) \tan (\theta(u(j))) \\
= & \|u(i)\|\|u(j)\| \cos (\theta(u(i))+\theta(u(j)))+\tan (\theta(u(i))) \tan (\theta(u(j))) \\
= & \frac{\cos (\theta(u(i))) \cos (\theta(u(j)))-\sin (\theta(u(i))) \sin (\theta(u(j)))}{\cos (\theta(u(i))) \cos (\theta(u(j)))} \\
& +\frac{\sin (\theta(u(i))) \sin (\theta(u(j)))}{\cos (\theta(u(i))) \cos (\theta(u(j)))} \\
=1, &
\end{array}
$$

since $\|u(i)\|=1 / \cos (\theta(u(i)))$ and $\|u(j)\|=1 / \cos (\theta(u(j)))$.

If $i j \notin E(G)$, then the angle between $u(i)$ and $u(j)$ is strictly more than $\theta(u(i))+$ $\theta(u(j))$ and similar calculations in this case demonstrate that $f(i)^{\mathrm{T}} f(j)<1$. 


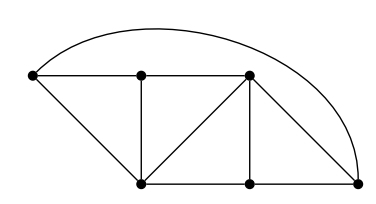

Figure 2: A graph $G^{\prime}$ with $\nu\left(G^{\prime}\right)=4$ and dot product dimension 3 .

Now Proposition 5 and Koebe's Theorem together imply that the dot product dimension of any planar graph is at most 4.

Let us mention work by Kotlov, Lovász and Vempala [7] on a notion closely related to dot product dimension. As a way of studying the Colin de Verdiére parameter $\mu(G)[2,3]$, Kotlov et al. introduced the following parameter. Let $\nu(G)$ denote the smallest $k$ such that there exists a vector labelling $u: V(G) \rightarrow \mathbb{R}^{k}$ that satisfies the following:

(i) $u(i)^{\mathrm{T}} u(j)=1$ if $i j \in E(G)$;

(ii) $u(i)^{\mathrm{T}} u(j)<1$ if $i j \notin E(G)$ and $i \neq j$; and

(iii) if $X$ is a symmetric $n \times n$ matrix such that $X_{i j}=0$ for all $i j \notin E(G)$ and $X_{i i}=0$ for all $i$ and $\sum_{j} X_{i j} u(j)=0$ for all $i$, then $X=0$.

Kotlov et al. demonstrated a very close relationship between $\nu(G)$ and $\mu(\bar{G})$.

Clearly, every graph $G$ is a $\nu(G)$-dot product graph. However, because (i) asks for equality and because of the extra demand (iii), $G$ might also be a $k$-dot product graph for some $k<\nu(G)$. For example, the graph $G^{\prime}$ in Figure 2 is the smallest such that $\nu\left(G^{\prime}\right)=4[7]$, but it is not difficult to check that $G^{\prime}$ is a 3-dot product graph.

Using Koebe's Theorem, Kotlov et al. obtained the following.

Theorem 6 (Corollary 8.7, [7]) If $G$ is a planar graph, then $\nu(G) \leq 4$.

This implies that every planar graph is a 4-dot product graph. Kotlov et al. also considered several subclasses of planar graphs.

Theorem 7 (Corollaries 8.7 and 9.8, [7]) If $G$ is an outerplanar graph or a planar graph with girth at least 5 , then $\nu(G) \leq 3$.

This implies that outerplanar graphs and planar graphs with girth at least 5 have dot product dimension at most 3. It is already known that the trees which are not caterpillars have dot product dimension $3[12,4]$. This for instance shows that imposing a girth condition cannot lower the above dot product dimension bound for outerplanar graphs. Although the above theorem shows that a girth constraint of 5 for planar graphs lowers the dot product dimension bound to 3 , there is not much hope of further improvement here. In Section 5, we show an example of a planar graph with girth 4 that is not a 3 -dot 
product graph. It is left open to determine whether there is any triangle-free planar graph without adjacent 4-cycles that has dot product dimension 4 .

We remark here that Reiterman et al. [13] studied the problem of bounding the dot product dimension for (not necessarily planar) graphs of prescribed girth; more generally, they showed that graphs of order $n$ and maximum average degree $\delta$ have dot product dimension at most $O\left(\delta^{2} \log n\right)$.

\section{A small planar graph of dimension 4}

In this section, we exhibit a small example $\hat{G}$ of a planar graph that has dot product dimension greater than 3. Let $\hat{G}$ be defined as follows. The vertex set $V(\hat{G})$ is defined as the union of the following sets:

$$
\bigcup_{i \in\{1, \ldots, 4\}}\left\{t_{i}, s_{i}, s_{-i}\right\} \text { and } \bigcup_{i, j \in\{1, \ldots, 4\}, i<j}\left\{s_{i j}\right\} .
$$

So there are eighteen vertices in total. The edge set $E(\hat{G})$ is defined as the union of the following sets:

$$
\begin{array}{r}
\bigcup_{i \in\{1, \ldots, 4\}}\left(\left\{s_{i} t_{i}\right\} \cup\left\{s_{-i} t_{j}: j \in\{1, \ldots, 4\} \backslash\{i\}\right\}\right) \quad \text { and } \\
\bigcup_{i, j \in\{1, \ldots, 4\}, i<j}\left\{t_{i} t_{j}, s_{i j} t_{i}, s_{i j} t_{j}\right\} .
\end{array}
$$

An embedding of $\hat{G}$ in the plane is depicted in Figure 3. We remark that $\hat{G}$ contains several copies of the complete graph $K_{4}$ on four vertices.

Suppose, to the contrary, that $u$ is a 3 -dot product representation of $\hat{G}$.

Four vectors in $\mathbb{R}^{3}$ are necessarily linearly dependent, so let $\lambda_{1}, \ldots, \lambda_{4}$ be scalars, not all zero, such that

$$
\mathbf{0}^{\mathrm{T}}=\lambda_{1} u\left(t_{1}\right)^{\mathrm{T}}+\lambda_{2} u\left(t_{2}\right)^{\mathrm{T}}+\lambda_{3} u\left(t_{3}\right)^{\mathrm{T}}+\lambda_{4} u\left(t_{4}\right)^{\mathrm{T}} .
$$

We will split the analysis into cases depending on how many of the scalars are nonnegative. By symmetry, there are only three cases to consider.

(i) All of the scalars are non-negative. Taking the inner product with $u\left(t_{1}\right)$ on both sides of (3), we obtain

$$
\begin{aligned}
0 & =0^{\mathrm{T}} u\left(t_{1}\right) \\
& =\lambda_{1} u\left(t_{1}\right)^{\mathrm{T}} u\left(t_{1}\right)+\lambda_{2} u\left(t_{2}\right)^{\mathrm{T}} u\left(t_{1}\right)+\lambda_{3} u\left(t_{3}\right)^{\mathrm{T}} u\left(t_{1}\right)+\lambda_{4} u\left(t_{4}\right)^{\mathrm{T}} u\left(t_{1}\right) \\
& \geq \lambda_{1}\left\|u\left(t_{1}\right)\right\|^{2}+\lambda_{2}+\lambda_{3}+\lambda_{4} \\
& >0
\end{aligned}
$$

where the first inequality holds since the vertex $t_{1}$ is adjacent to $t_{2}, t_{3}$ and $t_{4}$, and the second inequality holds since not all of the scalars are zero. We have reached a contradiction. 


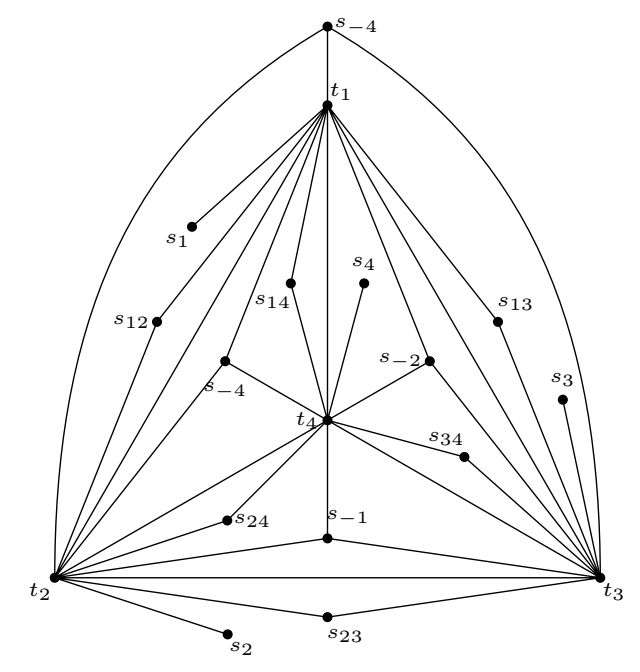

Figure 3: A planar graph $\hat{G}$ with dot product dimension 4 .

(ii) Exactly three of the scalars are non-negative. Without loss of generality, let us assume $-\lambda_{4}>0$ and hence $\lambda_{1}, \lambda_{2}, \lambda_{3} \geq 0$. We rewrite (3) as

$$
-\lambda_{4} u\left(t_{4}\right)^{\mathrm{T}}=\lambda_{1} u\left(t_{1}\right)^{\mathrm{T}}+\lambda_{2} u\left(t_{2}\right)^{\mathrm{T}}+\lambda_{3} u\left(t_{3}\right)^{\mathrm{T}} .
$$

If we take the inner product with $u\left(s_{-4}\right)$ on both sides of $(4)$, we obtain

$$
\begin{aligned}
-\lambda_{4} & >-\lambda_{4} u\left(t_{4}\right)^{\mathrm{T}} u\left(s_{-4}\right) \\
& =\lambda_{1} u\left(t_{1}\right)^{\mathrm{T}} u\left(s_{-4}\right)+\lambda_{2} u\left(t_{2}\right)^{\mathrm{T}} u\left(s_{-4}\right)+\lambda_{3} u\left(t_{3}\right)^{\mathrm{T}} u\left(s_{-4}\right) \\
& \geq \lambda_{1}+\lambda_{2}+\lambda_{3}
\end{aligned}
$$

where the first inequality holds since $t_{4}$ and $s_{-4}$ are not adjacent and the second inequality holds since $s_{-4}$ is adjacent to $t_{1}, t_{2}$ and $t_{3}$. However, if we take the inner product with $u\left(s_{4}\right)$ on both sides of (4) instead, we obtain

$$
\begin{aligned}
-\lambda_{4} & \leq-\lambda_{4} u\left(t_{4}\right)^{\mathrm{T}} u\left(s_{4}\right) \\
& =\lambda_{1} u\left(t_{1}\right)^{\mathrm{T}} u\left(s_{4}\right)+\lambda_{2} u\left(t_{2}\right)^{\mathrm{T}} u\left(s_{4}\right)+\lambda_{3} u\left(t_{3}\right)^{\mathrm{T}} u\left(s_{4}\right) \\
& <\lambda_{1}+\lambda_{2}+\lambda_{3},
\end{aligned}
$$

where the first inequality holds since $t_{4}$ and $s_{4}$ are adjacent and the second inequality holds since $s_{4}$ is adjacent to $t_{1}, t_{2}$ and $t_{3}$. Again, we have reached a contradiction.

(iii) Exactly two of the scalars are non-negative. Without loss of generality, assume $-\lambda_{3},-\lambda_{4}>0$, and hence $\lambda_{1}, \lambda_{2} \geq 0$. We rewrite (3) as

$$
-\lambda_{3} u\left(t_{3}\right)^{\mathrm{T}}-\lambda_{4} u\left(t_{4}\right)^{\mathrm{T}}=\lambda_{1} u\left(t_{1}\right)^{\mathrm{T}}+\lambda_{2} u\left(t_{2}\right)^{\mathrm{T}} .
$$


If we take the inner product with $u\left(s_{12}\right)$ on both sides of $(5)$, we obtain

$$
\begin{aligned}
-\lambda_{3}-\lambda_{4} & >-\lambda_{3} u\left(t_{3}\right)^{\mathrm{T}} u\left(s_{12}\right)-\lambda_{4} u\left(t_{4}\right)^{\mathrm{T}} u\left(s_{12}\right) \\
& =\lambda_{1} u\left(t_{1}\right)^{\mathrm{T}} u\left(s_{12}\right)+\lambda_{2} u\left(t_{2}\right)^{\mathrm{T}} u\left(s_{12}\right) \geq \lambda_{1}+\lambda_{2},
\end{aligned}
$$

where the first inequality holds since $s_{12}$ is neither adjacent to $t_{3}$ nor $t_{4}$ and the second inequality holds since $s_{12}$ is adjacent to $t_{1}$ and $t_{2}$. On the other hand, if we take the inner product with $u\left(s_{34}\right)$ on both sides of $(5)$, we obtain

$$
\begin{aligned}
-\lambda_{3}-\lambda_{4} & \leq-\lambda_{3} u\left(t_{3}\right)^{\mathrm{T}} u\left(s_{12}\right)-\lambda_{4} u\left(t_{4}\right)^{\mathrm{T}} u\left(s_{12}\right) \\
& =\lambda_{1} u\left(t_{1}\right)^{\mathrm{T}} u\left(s_{12}\right)+\lambda_{2} u\left(t_{2}\right)^{\mathrm{T}} u\left(s_{12}\right)<\lambda_{1}+\lambda_{2},
\end{aligned}
$$

where the first inequality holds since $s_{34}$ is adjacent to $t_{3}$ and $t_{4}$ and the second inequality holds since $s_{34}$ is neither adjacent to $t_{1}$ nor $t_{2}$. This is a contradiction.

In all cases, we have reached a contradiction. (One scalar non-negative is the same as three non-negative: just multiply $(3)$ by -1 . The case in which none of the scalars is

non-negative can be dealt with in the same way.) This concludes the proof that $\hat{G}$ is not a 3-dot product graph.

\section{A triangle-free planar graph of dimension 4}

In this section, we provide an alternative example $H_{k}$ of a planar graph with dot product dimension greater than 3 that satisfies the additional property that it does not contain a triangle as a subgraph.

We construct graphs $F, G, H$ as follows.

(i) We start with $K_{4}$, the complete graph on the four vertices $t_{1}, t_{2}, t_{3}, t_{4}$.

(ii) To obtain $F$, we subdivide each edge $t_{i} t_{j}$ of $K_{4}$ five times; that is, we replace $t_{i} t_{j}$ by a path $P_{i j}=t_{i} t_{i j}^{(1)} \ldots t_{i j}^{(5)} t_{j}$ with five internal vertices.

(iii) The graph $F$ divides the plane into four faces. To obtain $G$, we place an additional vertex inside each face of $F$. Here $f_{i}$ will denote the vertex in the face whose limiting cycle does not contain $t_{i}$. We connect $f_{i}$ to $t_{j}$ for $j \neq i$ and to $t_{j k}^{(2)}$ and $t_{j k}^{(4)}$ for $j, k \neq i$.

(iv) Finally, to obtain $H$ we attach four leaves to each vertex of $G$.

The graphs $F, G$ and $H$ are depicted in Figure 4 . For $k \in \mathbb{N}$, let the graph $H_{k}$ consist of $k$ disjoint copies of $H$. Clearly, $H_{k}$ is planar and triangle-free for all $k$. In the rest of this section we shall prove the following.

Theorem 8 The graph $H_{k}$ is not a 3 -dot product graph for some $k \in \mathbb{N}$.

Proof: The proof is by contradiction. Let us assume that $H_{k}$ has a 3 -dot product representation for all $k$. Then the following must hold. 

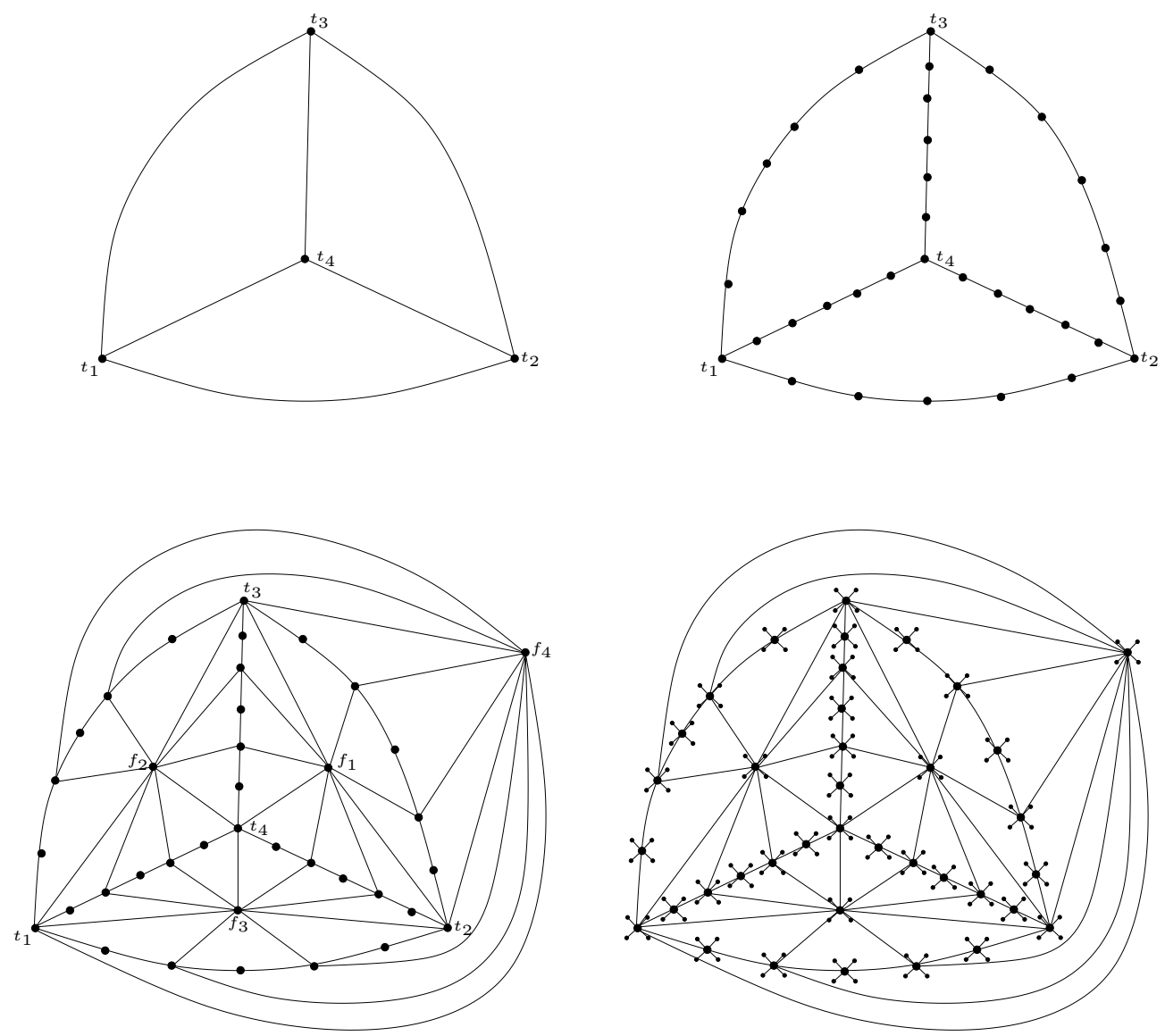

Figure 4: The graphs $K_{4}$ (top left), $F$ (top right), $G$ (bottom left) and $H$ (bottom right).

Claim 9 For every $\eta>0$, there is a 3-dot product representation $u$ of $H$, with $\|u(t)\|<$ $1+\eta$ for all $t \in V(H)$.

Proof of Claim 9: Suppose there is no such representation. Then there must exist a constant $\eta>0$ such that in every 3-dot product representation $u: V(H) \rightarrow \mathbb{R}^{3}$ of $H$, there is a vertex $t \in V(H)$ such that $\|u(t)\| \geq 1+\eta$.

As $H_{k}$ is the disjoint union of $k$ copies of $H$, in any 3-dot product representation $u: V\left(H_{k}\right) \rightarrow \mathbb{R}^{3}$, there is a vertex in each of the $k$ copies of $H$ whose corresponding vector has length at least $1+\eta$. Let $s_{1}, \ldots, s_{k}$ denote these vertices. We know $s_{i} s_{j} \notin E\left(H_{k}\right)$ for all $1 \leq i<j \leq k$, and $\left\|u\left(s_{i}\right)\right\| \geq 1+\eta$ for all $1 \leq i \leq k$. have

Let us write $v\left(s_{i}\right):=u\left(s_{i}\right) /\left\|u\left(s_{i}\right)\right\|$ and $l\left(s_{i}\right):=\left\|u\left(s_{i}\right)\right\|$. As $u\left(s_{i}\right)^{\mathrm{T}} u\left(s_{j}\right)<1$, we must

$$
v\left(s_{i}\right)^{\mathrm{T}} v\left(s_{j}\right)<\frac{1}{l\left(s_{i}\right) l\left(s_{j}\right)} \leq(1+\eta)^{-2} .
$$

Write $\rho:=\arccos \left((1+\eta)^{-2}\right)$. Note that $\operatorname{dist}\left(v\left(s_{i}\right), v\left(s_{j}\right)\right)>\rho$ for all $1 \leq i<j \leq k$. Hence the spherical caps $\operatorname{cap}\left(v\left(s_{1}\right), \rho / 2\right), \ldots, \operatorname{cap}\left(v\left(s_{k}\right), \rho / 2\right)$ must be disjoint subsets of 
the sphere. These caps all have the same area, which depends only on $\rho$, and hence only on $\eta$. Let us denote this area by $f(\eta)$. It is possible to express $f(\eta)$ explicitly in terms of $\eta$, but there is no need to do this here. We get

$$
4 \pi=\operatorname{area}\left(S^{2}\right) \geq \sum_{i=1}^{k} \operatorname{area}\left(\operatorname{cap}\left(v\left(s_{i}\right), \rho / 2\right)\right)=k \cdot f(\eta),
$$

which is impossible if we choose $k>4 \pi / f(\eta)$.

Let us fix a small $\eta$ (say $\eta:=1 / 10^{10}$ ) and let $u: V(H) \rightarrow \mathbb{R}^{3}$ be a representation provided by Claim 9. For $s \in V(H)$ let us write $l(s):=\|u(s)\|, v(s):=u(s) /\|u(s)\|$. Let us observe that

$$
s t \in E(H) \quad \text { if and only if } \quad v(s)^{\mathrm{T}} v(t) \geq 1 / l(s) l(t) .
$$

Note also that each spherical arc on $S^{2}$ corresponding to an edge in $H$ has length at most $\rho=\arccos \left((1+\eta)^{-2}\right)$.

Claim 10 We can assume without loss of generality that no three of $\{v(s): s \in V(H)\}$ lie on the same great circle/equator of $S^{2}$.

Proof of Claim 10: Clearly it suffices to show that without loss of generality no three of $\{u(s): s \in V(H)\}$ lie on the same linear space of dimension two. Let $u: V(H) \rightarrow \mathbb{R}^{3}$ be an arbitrary 3-dot product representation of $H$ such that $\|u(s)\|<1+\eta$ for all $s \in V(H)$. Then we can find a $\xi>0$ such that $(1+\xi)\|u(s)\|<1+\eta$ for all $s$, and $(1+\xi)^{2} u(s)^{T} u(t)<1$ for all non-edges $s t \notin E(H)$. Thus $u^{\prime}:=(1+\xi) u$ is also a valid 3-dot product representation, such that $u^{\prime}(s)^{T} u^{\prime}(t)<1$ for all non-edges $s t \notin E(H)$ and $u^{\prime}(s)^{T} u^{\prime}(t)>1$ for all edges $s t \in E(H)$. We can now add very small perturbations to $u^{\prime}$ to obtain a valid 3-dot product representation $u^{\prime \prime}$ such that no three vectors lie in the same linear hyperplane, and still $u^{\prime \prime}(s)<1+\eta$ for all $s \in V(H)$.

For the remainder of the proof, we make the assumption that no three of $\{v(s): s \in$ $V(H)\}$ lie on the same great circle.

Recall that $G \subseteq H$ is the subgraph induced by all non-leaf vertices.

Claim 11 For every $t \in V(G)$, we have $l(t)>1$.

Proof of Claim 11: Suppose that some $s \in V(G)$ satisfies $l(s) \leq 1$. Let $s_{1}, s_{2}, s_{3}, s_{4}$ be the four leaves attached to $s$, in clockwise order. One of the angles $\angle s_{1} s s_{2}, \angle s_{2} s s_{3}$, $\angle s_{3} s s_{4}, \angle s_{4} s s_{1}$ must be at most $\pi / 2$, since their sum is at most $2 \pi$.

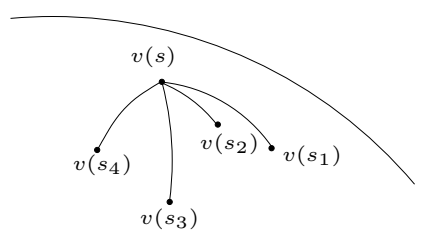

THE ELECTRONIC JOURNAL OF COMBINATORICS 18 (2011), \#P216 
Without loss of generality, we may assume it is $\gamma:=\angle s_{1} s s_{2}$. We have $\cos (\gamma) \geq 0$, so that the spherical cosine rule (2) gives that

$$
\begin{aligned}
v\left(s_{1}\right)^{\mathrm{T}} v\left(s_{2}\right) & \geq\left(v(s)^{\mathrm{T}} v\left(s_{1}\right)\right) \cdot\left(v(s)^{\mathrm{T}} v\left(s_{2}\right)\right) \\
& \geq\left(\frac{1}{l(s) l\left(s_{1}\right)}\right)\left(\frac{1}{l(s) l\left(s_{2}\right)}\right) \geq \frac{1}{l\left(s_{1}\right) l\left(s_{2}\right)},
\end{aligned}
$$

using the property that $l(s) \leq 1$ for the last inequality. But then we must have $s_{1} s_{2} \in$ $E(H)$, a contradiction.

Claim 12 Suppose that st, $s^{\prime} t^{\prime} \in E(G)$ are edges with $s, s^{\prime}, t, t^{\prime}$ distinct and suppose that the $\operatorname{arcs}[v(s), v(t)]$ and $\left[v\left(s^{\prime}\right), v\left(t^{\prime}\right)\right]$ cross. Then at least one of $s s^{\prime}, s t^{\prime}, t s^{\prime}, t t^{\prime}$ is also an edge of $G$.

Proof of Claim 12: Suppose that the arcs $[v(s), v(t)]$ and $\left[v\left(s^{\prime}\right), v\left(t^{\prime}\right)\right]$ cross and that $s s^{\prime}, s t^{\prime}, t s^{\prime}, t t^{\prime} \notin E(G)$. Consider the angles of the spherical 4-gon with corners $v(s), v\left(s^{\prime}\right)$, $v(t), v\left(t^{\prime}\right)$. Since the sum of the angles of a spherical 4 -gon is larger than $2 \pi$, at least one angle is larger than $\pi / 2$. We may assume without loss of generality that the points are in clockwise order $v(s), v\left(s^{\prime}\right), v(t), v\left(t^{\prime}\right)$ and that the angle $\angle v\left(s^{\prime}\right) v(s) v\left(t^{\prime}\right)$ is more than $\pi / 2$.

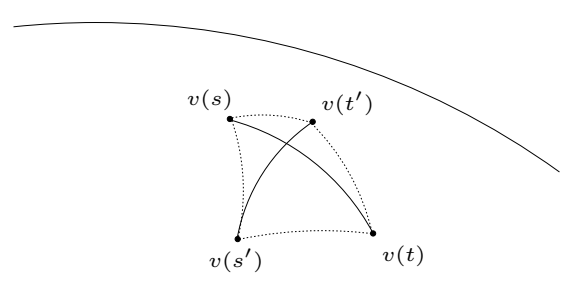

Then by version (2) of the spherical cosine rule we must have

$$
v\left(s^{\prime}\right)^{\mathrm{T}} v\left(t^{\prime}\right)<\left(v(s)^{\mathrm{T}} v\left(s^{\prime}\right)\right)\left(v(s)^{\mathrm{T}} v\left(t^{\prime}\right)\right) .
$$

Since $l(s) \geq 1$ by Claim 11 and $s s^{\prime}, s t^{\prime} \notin E(G)$, this gives

$$
v\left(s^{\prime}\right)^{\mathrm{T}} v\left(t^{\prime}\right)<\left(\frac{1}{l(s) l\left(s^{\prime}\right)}\right)\left(\frac{1}{l(s) l\left(t^{\prime}\right)}\right) \leq \frac{1}{l\left(s^{\prime}\right) l\left(t^{\prime}\right)},
$$

which contradicts $s^{\prime} t^{\prime} \in E(G)$.

If $s_{1}, s_{2}, s_{3} \in V(G)$, then $v\left(s_{1}\right), v\left(s_{2}\right), v\left(s_{3}\right)$ determine a spherical triangle $\Delta=$ $\left[v\left(s_{1}\right), v\left(s_{2}\right)\right] \cup\left[v\left(s_{2}\right), v\left(s_{3}\right)\right] \cup\left[v\left(s_{1}\right), v\left(s_{3}\right)\right]$ (which by Claim 12 is "non-degenerate"). Then $\Delta \subseteq \operatorname{cap}\left(v_{1}, 100 \rho\right)$ because each spherical arc corresponding to an edge of $G$ has length at most $\rho$ and $G$ has diameter at most 101. Note that $S^{2} \backslash \Delta$ consists of two path-connected components by the Jordan curve theorem. As $\rho$ is very small, exactly one of these components has area bigger than $3.9 \pi$. We shall refer to this component as the "outside" of $\Delta$, and the other component as the "inside". 
Claim 13 Suppose that $s_{1} s_{2} s_{3}$ form a path in $G$, and $v(s)$ lies inside the spherical triangle determined by $v\left(s_{1}\right), v\left(s_{2}\right), v\left(s_{3}\right)$. Then either $s s_{1} \in E(G)$ or $s s_{2} \in E(G)$ or $s s_{3} \in E(G)$.

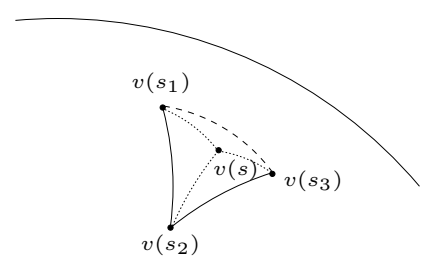

Proof of Claim 13: Observe that if $v(s)$ lies inside the (smaller of the two) spherical triangle(s) defined by $v\left(s_{1}\right), v\left(s_{2}\right), v\left(s_{3}\right)$, then we must have

$$
\angle v\left(s_{1}\right) v(s) v\left(s_{2}\right)+\angle v\left(s_{2}\right) v(s) v\left(s_{3}\right)+\angle v\left(s_{1}\right) v(s) v\left(s_{3}\right)=2 \pi .
$$

This shows that one of $\angle v\left(s_{1}\right) v(s) v\left(s_{2}\right), \angle v\left(s_{1}\right) v(s) v\left(s_{3}\right)$ is at least $\pi / 2$. (Otherwise we would have $\angle v\left(s_{1}\right) v(s) v\left(s_{3}\right)>\pi$, which is impossible.) Without loss of generality, we may assume that $\angle v\left(s_{1}\right) v(s) v\left(s_{2}\right) \geq \pi / 2$. The spherical cosine rule (2) gives that

$$
v\left(s_{1}\right)^{\mathrm{T}} v\left(s_{2}\right) \leq\left(v(s)^{\mathrm{T}} v\left(s_{1}\right)\right)\left(v(s)^{\mathrm{T}} v\left(s_{2}\right)\right) .
$$

If both $s s_{1}, s s_{2} \notin E(G)$, then $v(s)^{\mathrm{T}} v\left(s_{1}\right)<1 /\left(l(s) l\left(s_{1}\right)\right)$ and $v(s)^{\mathrm{T}} v\left(s_{2}\right)<1 /\left(l(s) l\left(s_{2}\right)\right)$. But then, as $l(s)>1$, we get from $(6)$ that $v\left(s_{1}\right)^{\mathrm{T}} v\left(s_{2}\right)<1 /\left(l\left(s_{1}\right) l\left(s_{2}\right)\right)$. This contradicts that $s_{1} s_{2} \in E(G)$.

Let $\gamma_{i j}$ denote the curve

$$
\gamma_{i j}:=\bigcup_{k=1}^{4}\left[v\left(t_{i j}^{(k)}\right), v\left(t_{i j}^{(k+1)}\right)\right]
$$

(i.e. $\gamma_{i j}$ is the union of the arcs corresponding to the internal edges of $P_{i j}$ ). By Claim 12, the curves $\gamma_{i j}$ and $\gamma_{k l}$ do not cross if $\{i, j\} \neq\{k, l\}$.

Let $C$ denote the cycle $P_{12} \cup P_{23} \cup P_{13}$. Let $\gamma$ denote the corresponding spherical polygon, i.e.

$$
\gamma:=\bigcup_{s t \in E(C)}[v(s), v(t)]
$$

As earlier with spherical triangles, we have $\gamma \subseteq \operatorname{cap}\left(v_{1}, 100 \rho\right)$. Also note that now $S^{2} \backslash \gamma$ consists of at least two path-connected components (by the Jordan curve theorem). Again, since $\rho$ is very small, exactly one of these components has area bigger than $3.9 \pi$. We shall refer to this component as the outside of $\gamma$, and the union of the other components as the inside of $\gamma$.

Claim 14 We may assume without loss of generality that $v\left(t_{4}\right)$ lies inside $\gamma$. 


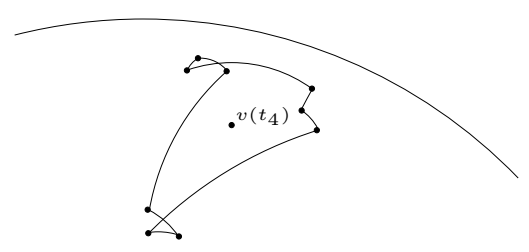

Proof of Claim 14: For $i \in\{1,2,3,4\}$, let $T_{i} \subseteq S^{2}$ denote the union of arcs

$$
T_{i}:=\bigcup_{s \in N\left(t_{i}\right)}\left[v\left(t_{i}\right), v(s)\right]
$$

Observe that by Claim 12 the $T_{i}$ 's are disjoint. Let $I_{i j} \subseteq \gamma_{i j}$ denote the minimal subset ("the minimal subcurve") that connects $T_{i}$ and $T_{j}$. Then the $I_{i j}$ s are disjoint, $I_{i j}$ hits each of $T_{i}, T_{j}$ in exactly one point, and $I_{i j}$ does not intersect $T_{k}$ for $k \neq i, j$.
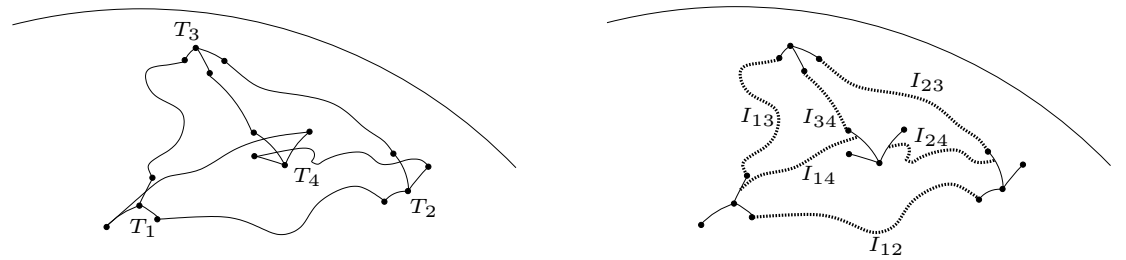

$S^{2} \backslash\left(T_{1} \cup I_{12} \cup T_{2} \cup I_{23} \cup T_{3} \cup I_{13}\right)$ consists of two regions and since $T_{4}$ does not intersect $T_{1} \cup I_{12} \cup T_{2} \cup I_{23} \cup T_{3} \cup I_{13}$ it must be contained in one of those regions. Applying a suitable relabelling if necessary, we can assume that $T_{4}$ lies inside the smaller of the two regions of $S^{2} \backslash\left(T_{1} \cup I_{12} \cup T_{2} \cup I_{23} \cup T_{3} \cup I_{13}\right)$. (This can for instance be see from the fact that $\bigcup_{i} T_{i} \cup \bigcup_{i j} I_{i j}$ contains a planar drawing of $K_{4}$.) But in that case $v\left(t_{4}\right) \in T_{4}$ lies inside $P$ as desired.

Claim $15 v\left(t_{4}\right)$ lies inside the spherical triangle determined by $v\left(f_{4}\right)$ and two consecutive points on $\gamma$.

Proof of Claim 15: Notice that no matter where $v\left(f_{4}\right)$ lies exactly the great circle through $v\left(t_{4}\right)$ and $v\left(f_{4}\right)$ hits $\gamma$ at least twice, and we can speak of the first segment of $P$ that lies behind $v\left(t_{4}\right)$ viewed from $v\left(f_{4}\right)$.
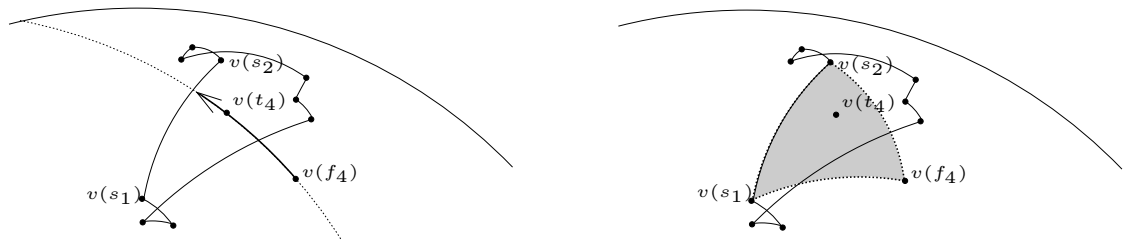

If the vertices corresponding to the endpoints of this segment are $v\left(s_{1}\right)$ and $v\left(s_{2}\right)$, then the spherical triangle determined by $v\left(f_{4}\right), v\left(s_{1}\right), v\left(s_{2}\right)$ clearly contains $v\left(t_{4}\right)$. By Claim 10, $v\left(t_{4}\right)$ cannot lie on the boundary of this spherical triangle.

Since $f_{4}, s_{1}, s_{2}$ induce a path in $G$, Claim 13 together with Claim 15 implies that $t_{4}$ must be adjacent to at least one of them. This is a contradiction since $t_{4}$ is neither adjacent to $f_{4}$ nor to any vertex of $C$. This concludes the proof of Theorem 8 . 


\section{References}

[1] N. Alon, P. Frankl, and V. Rödl. Geometrical realization of set systems and probabilistic communication complexity. In Proc. 26th Annual Symp. on Foundations of Computer Science (FOCS), Portland, Oregon, IEEE, pages 277-280, 1985.

[2] Y. Colin de Verdière. Sur un nouvel invariant des graphes et un critère de planarité. J. Combin. Theory Ser. B, 50(1):11-21, 1990.

[3] Y. Colin de Verdière. On a new graph invariant and a criterion for planarity. In Graph structure theory (Seattle, WA, 1991), volume 147 of Contemp. Math., pages 137-147. Amer. Math. Soc., Providence, RI, 1993.

[4] C. M. Fiduccia, E. R. Scheinerman, A. Trenk, and J. S. Zito. Dot product representations of graphs. Discrete Math., 181(1-3):113-138, 1998.

[5] R. J. Kang and T. Müller. Dot product representations of planar graphs. In U. Brandes and S. Cornelsen, editors, Graph Drawing, volume 6502 of Lecture Notes in Computer Science, pages 287-292. Springer, 2010.

[6] P. Koebe. Kontaktprobleme der Konformen Abbildung. Berichte über die Verhandlungen der Sächsischen Akademie der Wissenschaften zu Leipzig, Math.-Phys. Klasse, 88:141-164, 1936.

[7] A. Kotlov, L. Lovász, and S. Vempala. The Colin de Verdière number and sphere representations of a graph. Combinatorica, 17(4):483-521, 1997.

[8] L. Lovász. On the Shannon capacity of a graph. IEEE Trans. Inform. Theory, 25(1):1-7, 1979.

[9] N. V. R. Mahadev and U. N. Peled. Threshold graphs and related topics, volume 56 of Annals of Discrete Math. North-Holland Publishing Co., Amsterdam, 1995.

[10] R. Paturi and J. Simon. Probabilistic communication complexity. J. Comput. System Sci., 33(1):106-123, 1986. Twenty-fifth annual symposium on foundations of computer science (Singer Island, Fla., 1984).

[11] J. Reiterman, V. Rödl, and E. Šiňajová. Embeddings of graphs in Euclidean spaces. Discrete Comput. Geom., 4(4):349-364, 1989.

[12] J. Reiterman, V. Rödl, and E. Šiňajová. Geometrical embeddings of graphs. Discrete Math., 74(3):291-319, 1989.

[13] J. Reiterman, V. Rödl, and E. Šiňajová. On embedding of graphs into Euclidean spaces of small dimension. J. Combin. Theory Ser. B, 56(1):1-8, 1992. 